\title{
Clinical Factors Related to Suspected Second Primary Lung Cancer Development in Patients with Head and Neck Cancer
}

\author{
Eui Bae Kim, M.D. ${ }^{1}$, Yong Park, M.D. ${ }^{1}$, Seh Jong Park, M.D. ${ }^{1}$, Dae Sik Kim, M.D. ${ }^{1}$, Jee Won Kim, \\ M.D. ${ }^{1}$, Hee Yun Seo, M.D., Ph.D. ${ }^{1}$, Hwa Jung Sung, M.D., Ph.D. ${ }^{1}$, In Keun Choi, M.D., Ph.D. ${ }^{1}$, Kyong \\ Hwa Park, M.D., Ph.D. ${ }^{1}$, Sang Cheul Oh, M.D., Ph.D. ${ }^{1}$, Chul Won Choi, M.D., Ph.D. ${ }^{1}$, Byung Soo \\ Kim, M.D., Ph.D. ${ }^{1}$, Yeul Hong Kim, M.D., Ph.D. ${ }^{1}$, Jun Suk Kim M.D., Ph.D. ${ }^{1}$, Sang Won Shin, M.D., \\ Ph.D. ${ }^{1}$, Chul Yong Kim, M.D., Ph.D. ${ }^{2}$ and Kwang-Yoon Jung, M.D., Ph.D. ${ }^{3}$ \\ ${ }^{1}$ Division of Oncology/Hematology, Department of Internal Medicine, ${ }^{2}$ Department of Radiation Oncology and ${ }^{3}$ Otolaryngology, \\ Korea University College of Medicine, Seoul, Korea
}

Purpose: The rate of second primary lung cancer development for patients with head and neck cancer (HNC) has been noted. The aim of our study was to evaluate the incidence and clinical features of suspected second primary lung cancer that developed in patients with primary HNC.

Materials and Methods: We conducted a retrospective study of 469 patients who were newly diagnosed with HNC at the Korea University Medical Center between January 2000 and December 2006.

Results: A total of 469 patients were included ( 389 men and 80 women). Eighteen patients (3.8\%) had suspected second primary lung cancers. Statistically significant clinical variables for lung cancer development included the origin site for the primary HNC (oro-hypopharynx and lar-

\section{INTRODUCTION}

Head and neck cancers (HNC) represent about $6 \%$ of all cancers worldwide (1). The development of HNC is closely associated with tobacco and alcohol use (2). Although several treatment strategies have been developed for $\mathrm{HNC}$, the long-term survival rates have improved only minimally during the last several decades (3). In addition, patients with HNC are at risk for developing second primary malignancies at a rate of $4 \%$ to $7 \%$ per year (4). The most common sites for a second primary tumor development are the head and neck and the lung (5).

Approximately $1 \%$ of head and neck squamous cell carcinomas (SCCs) are also associated with synchronous lung cancers (6). A second primary lung cancer in conjunction with an

Correspondence: Sang Won Shin, Division of Oncology/Hematology, Department of Internal Medicine, Korea University Anam Hospital, Korea University College of Medicine, 126-1, Anam-dong 5 ga, Seongbuk-gu, Seoul 136-705, Korea. (Tel) 82-2-920-5848, (Fax) 82-2-922-5974, (E-mail) Sangwonshin@yahoo.com

Received October 14, 2008, Accepted November 11, 2008 ynx) ( $p=0.048)$, abnormal chest $x$-ray findings $(p=0.027)$ and the histological HNC type (squamous cell carcinoma) $(p=0.032)$. When the second primary lung cancers were combined with HNCs, the adjusted overall survival of patients with a second primary lung cancer was 16 months ( $p<0.001)$.

Conclusions: Considering the relative risk factors for a second primary lung cancer developing in patients with HNC, advanced diagnostic tools, such as chest CT or PET CT scan, should be applied for the early detection of a second primary lung cancer. (Cancer Res Treat 2008;40:178-183)

Key Words: Chest x-ray, Head and neck neoplasms, Second primary cancer

$\mathrm{HNC}$ is related to poor survival due to the advanced stage at time of diagnosis (7). Hence, early detection of a second primary lung cancer may be important for prolonging the survival of patients with HNC. All head and neck cancer patients are routinely screened at initial diagnosis using chest $\mathrm{x}$-rays for early detection of a second primary lung cancer (8). Some clinicians will also perform comprehensive bronchoscopy, with or without bronchial washing, to evaluate the possibility of a second primary lung cancer (9).

No data are currently available to determine if lung cancer screening by computed tomography (CT) is useful for improving lung cancer mortality (10), although CT scans have recently been suggested for early detection of second primary lung cancers. Loh et al (11) reported that the detection rate for lung metastasis or synchronous lung primary tumor by CT was higher than for x-rays. Thus, chest CT scans are recommended for patients with newly diagnosed T4 head and neck SCC. Bayraktaroglu et al (12) evaluated the utility of CT imaging for the management of solitary pulmonary nodules. However, routine use of CT has significant disadvantages, including high cost (10). Therefore, chest CT scans must be judiciously employed for patients with risk factors for developing a second primary lung cancer.

The aims of our study were to evaluate the incidence and 
clinical risk factors associated with the development of a second primary lung cancer for patients with HNC. Our goal was to more clearly define the optimal approach for detecting second primary lung tumors for patients who were newly diagnosed with HNC.

\section{MATERIALS AND METHODS}

This was a retrospective study of 469 patients who were newly diagnosed with $\mathrm{HNC}$ at the Korea University Medical Center between January 2000 and December 2006.

\section{1) Eligibility criteria}

We enrolled patients with histologically confirmed primary HNC using the morphological codes recorded by the registries. These were based on the WHO Classification of Tumors (13). Patients were included if they had primary tumors that originated in the oral cavity, nasal cavity, paranasal sinus, nasopharynx, oro-hypopharynx, larynx or salivary gland. Patients who had been cured of other malignancies were eligible if these other malignancies were histologically different from the primary HNC. They were excluded if the primary tumor originated from the skin or if the cell type was lymphoma.

\section{2) Diagnostic criteria used to define a second primary lung cancer}

The criteria used to diagnose second primary neoplasia were as follows: the neoplasm was clearly malignant as determined by histology, each neoplasm was geographically separate and distinct, and the possibility that the second neoplasm resulted from metastasis could be excluded (14).

In the absence of definitive histological differences for both tumors, a second primary lung cancer had to be separated by more than $2 \mathrm{~cm}$ in the normal epithelium from the $\mathrm{HNC}$, it had to be solitary, and it had to have distinct imunohistochemical findings.

\section{3) Abstraction from records}

Patient medical records were reviewed for the following variables: age, sex, alcohol and tobacco use, site of origin, TNM stage, histology, treatment of initial primary or second primary cancers, synchronicity and initial chest x-ray findings. Information for alcohol and tobacco use was abstracted from the first medical record for each cancer. To facilitate the analysis, tobacco use was defined as smoking more than one pack-year or smoking for more than 5 years before detection of the primary cancer. Patients who had never smoked or drunk alcohol, or those who reported infrequent usage (a few times a year) were categorized as nonusers. $\mathrm{T}$ and $\mathrm{N}$ classifications were made according to the criteria of the American Joint Commission on Cancer (AJCC). Synchronicity was classified as either synchronous or metachronous. A synchronous primary tumor was diagnosed when the suspected second primary lung cancer was found within 6 months of the diagnosis of HNC. Suspected lung cancer detected more than 6 months after the diagnosis of HNC was classified as metachronous carcinoma (15). Chest abnormalities included nodules, opacities, granulomas, pleural adhesion or thickening, interstitial lung disease, emphysema, calcifications, atelectasis, inflammatory scars, bronchitis and bronchiectasis.

\section{4) Statistical analysis}

Depending on the data type, Fisher's exact test or Wilcoxon's rank sum test was used to test for differences in various characteristics of primary $\mathrm{HNC}$ and suspected second primary lung cancer. Overall survival was calculated from the time of diagnosis of primary HNC or suspected second primary lung cancer and was computed using a Kaplan-Meier survival curve. A p-value $<0.05$ was considered statistically significant. SPSS 12.0 (Statistical Package for the Social Sciences, release 12.0) was used for the statistical analysis.

\section{RESULTS}

There were 469 total patients in this study, including 80 female patients $(17.1 \%)$. Table 1 summarizes the demographics and baseline characteristics for this study population. Their median age was 67.0 years. The median follow-up period was 29.0 months. Most patients were smokers $(75.4 \%)$ or alcohol users $(70.7 \%)$. The sites of HNC origin were as follows: 69 tumors from the oral cavity (14.8\%), 26 from the salivary glands $(5.6 \%), 34$ from the nasal cavity or paranasal sinus $(7.2 \%), 49$ from the nasopharynx $(10.4 \%), 113$ from the oro-hypopharynx (24.0\%) and 178 from the larynx (38.0\%). The most common site of origin for HNC was the larynx. Approximately $57.4 \%$ of HNCs were advanced stage at initial diagnosis. Squamous cell carcinoma was the most common histologic type. About half of the patients $(55.4 \%)$ had normal $\mathrm{x}$-rays when they were initially diagnosed with HNC.

The development of suspected second primary lung cancer was confirmed in 18 patients $(3.8 \%)$ among the $469 \mathrm{HNC}$ patients (Table 1). The median age of these patients was 69.0 years, with only 1 female patient. The median follow-up period was 37.0 months. Most of these patients were smokers $(n=17)$ or alcohol users $(n=16)$. All suspected second primary lung cancers occurred in patients with HNC originating from the oro-hypopharynx or larynx. Most second primary lung cancers were discovered at an advanced stage $(n=12)$. Squamous cell carcinoma was the histological HNC type seen in all patients with suspected second primary lung cancers. Thirteen patients had at least 1 abnormal chest $\mathrm{x}$-ray finding at the time of HNC diagnosis.

Statistically significant factors for second primary lung cancer included the site of origin $(\mathrm{p}=0.048)$, histology $(\mathrm{p}=$ 0.032 ) and chest $x$-ray findings $(p=0.027)$ (Table 1$)$.

The suspected second primary lung cancers were confirmed as SCC in 11 patients, small cell lung cancer (SCLC) in 3 patients and undifferentiated non-small cell lung cancer (NSCLC) in 4 patients (Table 2). However, there were no patients with adenocarcinoma. Most suspected second primary lung cancers were detected more than 6 months after HNC was diagnosed. Only 3 patients were synchronously diagnosed with suspected second primary lung cancer and HNC. The stages for the suspected second primary lung cancers for these 18 patients were as follows: early stage (I $\sim$ II, limited) ( 7 cases) and advanced stage (III $\sim \mathrm{IV}$, extended) (11 cases). The overall 
Table 1. Demographics and baseline characteristics for the study population

\begin{tabular}{|c|c|c|c|c|}
\hline & $\begin{array}{c}\text { Total } \\
(\mathrm{n}=469)\end{array}$ & $\begin{array}{l}\text { Primary } \\
\text { HNC* } \\
\text { without } \\
\text { second } \\
\text { primary } \\
\text { lung cancer 1 } \\
(\mathrm{n}=451)\end{array}$ & $\begin{array}{l}\text { Primary } \\
\text { HNC* } \\
\text { with } \\
\text { second } \\
\text { primary } \\
\text { lung cancer } \\
(\mathrm{n}=18)\end{array}$ & p-value \\
\hline Sex & & & & $0.225^{\|}$ \\
\hline Male & $389(82.9 \%)$ & $372(95.6 \%)$ & $17(4.4 \%)$ & \\
\hline Female & $80(17.1 \%)$ & 79 (98.7\%) & $1(1.3 \%)$ & \\
\hline Age (years) & $67.0^{\pi}$ & $66.0 * *$ & $69.0^{\dagger \dagger}$ & $0.188^{\text {キキ }}$ \\
\hline Smoking & & & & $0.088^{\|}$ \\
\hline Yes & $354(75.4 \%)$ & $337(95.2 \%)$ & $17(4.8 \%)$ & \\
\hline No & $115(24.6 \%)$ & $114(99.1 \%)$ & $1(0.9 \%)$ & \\
\hline Alcohol & & & & $0.113^{\|}$ \\
\hline Yes & $332(70.7 \%)$ & $316(95.2 \%)$ & $16(4.8 \%)$ & \\
\hline No & $137(29.3 \%)$ & $135(98.5 \%)$ & $2(1.5 \%)$ & \\
\hline HNC Origin Site & & & & $0.048^{\|}$ \\
\hline Oral cavity & $69(14.8 \%)$ & $69(100 \%)$ & 0 & \\
\hline Salivary gland & $26(5.6 \%)$ & $26(100 \%)$ & 0 & \\
\hline $\begin{array}{l}\text { Nasal cavity and } \\
\text { paranasal sinus }\end{array}$ & $34(7.2 \%)$ & $34(100 \%)$ & 0 & \\
\hline Nasopharynx & $49(10.4 \%)$ & $49(100 \%)$ & 0 & \\
\hline Oro-hypopharynx & $113(24.0 \%)$ & $107(94.7 \%)$ & $6(5.3 \%)$ & \\
\hline Larynx & $178(38.0 \%)$ & $166(89.8 \%)$ & $12(10.2 \%)$ & \\
\hline Stage of HNC & & & & $0.474^{\|}$ \\
\hline $\mathrm{I} \sim \mathrm{II}$ & $200(42.6 \%)$ & 194 (97\%) & $6(3 \%)$ & \\
\hline III $\sim$ IV & $269(57.4 \%)$ & $257(95.5 \%)$ & $12(4.5 \%)$ & \\
\hline Histology of HNC* & & & & $0.032^{\|}$ \\
\hline $\mathrm{SCC}^{\dagger}$ & $372(79.3 \%)$ & $354(95.2 \%)$ & $18(4.8 \%)$ & \\
\hline Non-SCC ${ }^{\dagger}$ & $97(20.7 \%)$ & $97(100 \%)$ & 0 & \\
\hline $\mathrm{CXR}^{\S}$ & & & & $0.027^{\|}$ \\
\hline Normal & $260(55.4 \%)$ & $255(98.1 \%)$ & $5(1.9 \%)$ & \\
\hline Abnormal & $209(44.6 \%)$ & $196(93.8 \%)$ & $13(6.2 \%)$ & \\
\hline
\end{tabular}

*head and neck cancer, ${ }^{\dagger}$ squamous cell carcinoma, ${ }^{\dagger}$ non-squamous cell carcinoma, ${ }^{\S}$ chest $\mathrm{x}$-ray, "Fisher's exact test, "[56.2 to 72.7], ${ }^{* *}[56.0$ to 73.0$],{ }^{\dagger \dagger}[64.0$ to 72.2$],{ }^{\neq \dagger}$ Wilcoxon's rank sum test.

median survival time with suspected second primary lung cancer was 16 months (Table 2).

We performed subgroup analyses to evaluate the relationship between HNC and suspected second primary lung cancers (Table 3). We analyzed the lung cancer stages and the event free time (EFT) from the time of diagnosis of HNC to the discovery of suspected lung cancer according to the site of origin of the primary HNC. In patients with oro-hypopharyngeal cancer, the suspected second primary lung cancer was usually advanced. In contrast, in patients with laryngeal cancer, which is located closer to the lung, the development of suspected second primary lung cancer was unrelated to the lung cancer stage $(p=0.038)$. The median EFT was 30.5 months (31.0 months at oro-hypopharynx, 29.5 at larynx). There was no significant difference in median EFT between these two groups $(\mathrm{p}=0.606) \quad($ Table 3$)$.
Table 2. Second primary lung cancer characteristics

\begin{tabular}{lc}
\hline \multicolumn{1}{c}{ Second primary lung cancer } & Number \\
\hline Cell type & \\
NSCLC $^{*}$ & 15 \\
SCC $^{\dagger}$ & 11 \\
Undifferentiated NSCLC* $^{*}$ & 4 \\
SCLC $^{\dagger}$ & 3 \\
Synchronicity & 3 \\
Synchronous & 15 \\
Metachronous & \\
Stage & \\
NSCLC & \\
I $\sim$ II & 6 \\
III $\sim$ IV & 9 \\
SCLC & \\
$\quad$ Limited & 1 \\
Extended & 2 \\
Overall median survival time (months) & 16 \\
\end{tabular}

${ }^{*}$ non-small cell lung cancer, ${ }^{\dagger}$ squamous cell carcinoma, ${ }^{\dagger}$ small cell lung cancer.

Table 3. The relationship of second primary lung cancer to stage and EFT according to site of origin of primary $\mathrm{HNC}$

\begin{tabular}{|c|c|c|c|}
\hline \multirow[b]{2}{*}{$\begin{array}{l}\text { Site of origin } \\
\text { of } \mathrm{HNC}^{\star}\end{array}$} & \multicolumn{2}{|c|}{ Lung cancer } & \multirow[b]{2}{*}{$\underset{(\text { months) }}{\text { Median } \mathrm{EFT}^{\dagger}}$} \\
\hline & $\begin{array}{c}\text { Early } \\
\text { stage } \\
\left(\mathrm{I} \sim \mathrm{II}^{\dagger}\right. \\
\left.\text { limit }^{\S}\right)\end{array}$ & $\begin{array}{c}\text { Advanced } \\
\text { stage } \\
\left(\mathrm{IIII} \sim \mathrm{IV}^{\ddagger}\right. \\
\left.\text { extended }^{\S}\right)\end{array}$ & \\
\hline Oro-hypopharynx & 0 & 6 & 31.0 (13.2 to 65.5$)$ \\
\hline Larynx & 7 & 5 & 29.5 (7.5 to 54.7 ) \\
\hline Total & 7 & 11 & 30.5 \\
\hline $\mathrm{p}$-value & \multicolumn{2}{|c|}{0.038} & 0.606 \\
\hline
\end{tabular}

*head and neck cancer, ${ }^{\dagger}$ event free time from HNC to lung cancer, ${ }^{\dagger}$ stage of non small cell lung cancer, ${ }^{\S}$ stage of small cell lung cancer, "Wilcoxon's rank sum test.

Many suspected second primary lung cancers were discovered at an advanced stage, and the majority of these patients received only palliative treatments, such as chemotherapy and radiotherapy (13 patients). However, most primary HNCs were treated with curative therapies, including surgery (116 patients), radiotherapy (30 patients) and combined chemotherapy (254 patients) (Table 4).

By multivariate analysis of the clinical characteristics related to occurrence of suspected second primary lung cancer, no factors were found to be statistically significant. This was probably due to the small number of patients with suspected second primary lung cancers (Table 5).

As shown in Fig. 1, the median survival time was 81 months in the HNC group and 72 months in the suspected second primary lung cancer group $(p=0.430)$. However, when the EFT was excluded from the analysis for overall survival for 
Table 4. Treatments for primary HNCs and second primary lung cancers

\begin{tabular}{|c|c|c|c|c|c|c|c|}
\hline \multirow{2}{*}{ Site of origin of $\mathrm{HNC}^{*}$} & \multicolumn{4}{|c|}{$\mathrm{HNC}^{*}$} & \multicolumn{3}{|c|}{ Second primary lung cancer } \\
\hline & $\mathrm{Op}^{\dagger}$ & $\mathrm{RT}^{\dagger}$ & Combined $^{\S}$ & Palliative & $\mathrm{Op}^{\dagger}$ & Combined $\|$ & Palliative \\
\hline Oral cavity & 29 & 3 & 29 & 8 & 0 & 0 & 0 \\
\hline Salivary gland & 5 & 1 & 19 & 1 & 0 & 0 & 0 \\
\hline Nasal cavity and paranasal sinus & 5 & 3 & 22 & 4 & 0 & 0 & 0 \\
\hline Nasopharynx & 1 & 4 & 26 & 18 & 0 & 0 & 0 \\
\hline Oro/hypopharynx & 12 & 12 & 59 & 30 & 0 & 1 & 5 \\
\hline Larynx & 64 & 7 & 99 & 8 & 3 & 1 & 8 \\
\hline Total & 116 & 30 & 254 & 69 & 3 & 2 & 13 \\
\hline
\end{tabular}

*head and neck cancer, ${ }^{\dagger}$ operation, ${ }^{\ddagger}$ radiotherapy, ${ }^{\S}$ Op plus RT, Op plus chemotherapy, or RT plus chemotherapy, ${ }^{\|}$Op plus RT or Op plus chemotherapy.

Table 5. Multivariate analysis of clinical factors related to occurrence of second primary lung cancer

\begin{tabular}{lrrrrr}
\hline \multicolumn{1}{c}{ Clinical factor } & \multicolumn{1}{c}{$\mathrm{Co}^{*}$} & $\mathrm{SE}^{\dagger}$ & $\mathrm{OR}^{\ddagger}$ & $95 \% \mathrm{CI}^{\S}$ & $\mathrm{p}$-value \\
\hline Age & 0.008 & 0.008 & 1.008 & $0.992 \sim 1.024$ & 0.316 \\
Sex & -0.413 & 0.274 & 0.662 & $0.387 \sim 1.131$ & 0.361 \\
CXR abnormality & 0.222 & 0.206 & 1.248 & $0.834 \sim 1.869$ & 0.282 \\
Histology of HNC & 0.189 & 0.251 & 1.208 & $0.738 \sim 1.976$ & 0.452 \\
Origin site of HNC & -0.452 & 0.223 & 0.882 & $0.376 \sim 0.901$ & 0.055 \\
\hline
\end{tabular}

*coefficient, ${ }^{\dagger}$ standard error, ${ }^{\dagger}$ odds ratio, ${ }^{\S}$ confidence interval, " chest $\mathrm{x}$-ray, "head and neck cancer.
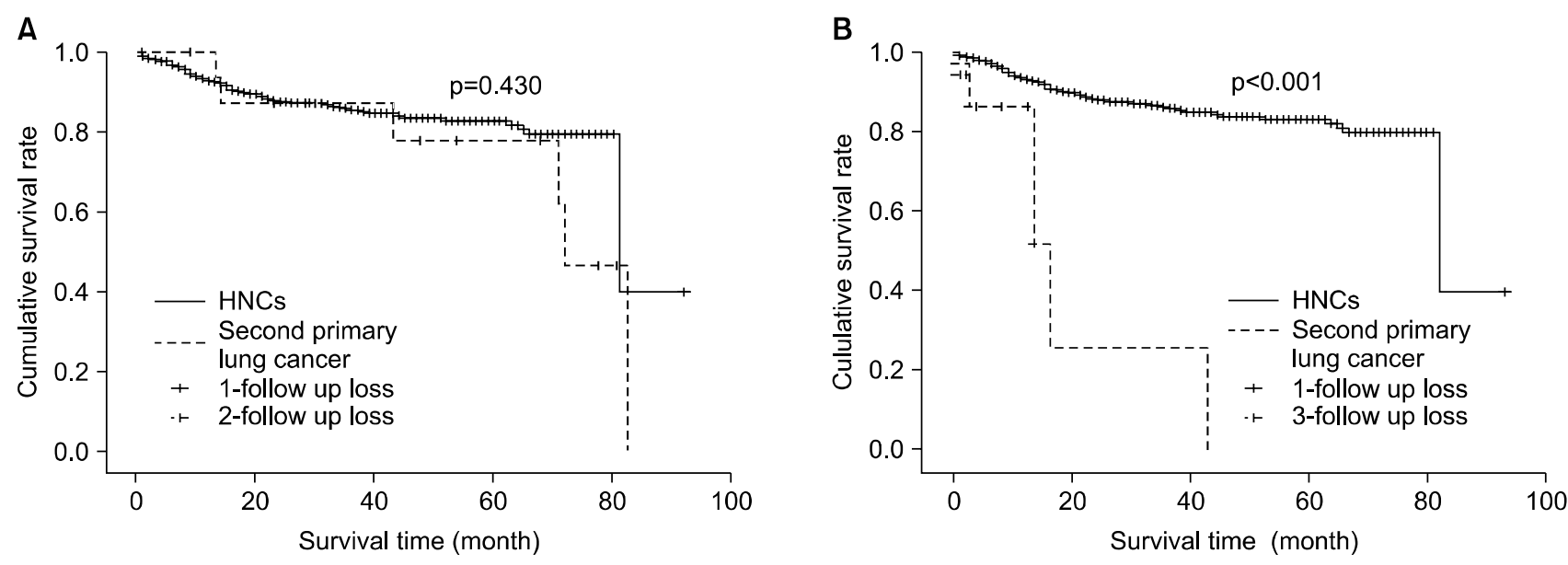

Fig. 1. Kaplan Meier survival curves for primary HNC and second primary lung cancer patients. (A) The median survival curves for HNC only patients and second primary lung cancer patients. (B) The adjusted median survival curves for HNC only patients and second primary lung cancer patients.

suspected second primary lung cancer, the adjusted median survival time for suspected second primary lung cancer was 16 months $(\mathrm{p}<0.001)$.

\section{DISCUSSION}

Billroth (17) first described the concept of several primary malignancies in the same patient in the 1860s. Warren and Gates (18) established the criteria for classifying multiple neo- plasms in 1932. In 1953, Slaughter et al (19) proposed the theory of "field cancerization" to explain the high incidence of secondary malignancies in environmentally-induced carcinomas, which referred to the continuous exposure of the upper aerodigestive tract epithelium to unknown carcinogenic agents. In 1999, Wu et al (20) proposed that carcinogenic agents, such as tobacco and alcohol, lead to genetic and histologic changes that result in synchronous or metachronous carcinoma development.

According to previous studies, the rates for synchronous or 
metachronous carcinoma development are between $8 \%$ and $21 \%$. Scelo et al (21) reported that the rate for second primary lung cancer development in patients with nasopharyngeal carcinoma ranges between $0.38 \%$ and $2.36 \%$. Hsieh et al (22) reported that the incidence of lung cancer in patients with upper aerodigestive tract cancer was $0.9 \%$. In the present study, the rate of synchronous or metachronous suspected lung cancer occurring in the presence of $\mathrm{HNC}$ was $3.8 \%$, which is higher than that reported by Hsieh et al.

Additionally, several interesting features were noted in our study. First, all suspected second primary lung cancers developed when the site of origin for the HNC was the oro-hypopharynx or larynx $(\mathrm{p}=0.048)$. The rate of suspected second primary lung cancer development was significantly higher in patients with oro-hypopharynx and larynx cancer compared with patients having HNCs arising from other sites of the head and neck. Similar risk factors, such as smoking and anatomical proximity of these areas to the lung, may contribute to this interesting finding.

We found that all suspected second primary lung cancers developed when the histological type of the primary HNC was SCC $(p=0.032)$. Therefore, when the histological type of a primary $\mathrm{HNC}$ is squamous cell, the clinician must consider the possibility of a second primary lung cancer and carefully follow-up with chest X-rays or chest CT scans.

We found that the majority of patients who developed a suspected second primary lung cancer already had at least 1 or more abnormal chest $\mathrm{x}$-ray findings when they were initially diagnosed with $\mathrm{HNC}$, and these findings were statistically significant $(\mathrm{p}=0.027)$. The abnormal chest $\mathrm{x}$-ray findings in patients with suspected second primary lung cancer were as follows: granuloma (2), indeterminate nodule (1), opacity (4), bronchitis (2), atelectasis (2), inflammatory scar change (1) and bronchiectasis (1). Suspected second primary lung cancers occurred in the same lobe in which the abnormal chest $\mathrm{x}$-ray findings were noted, except for 1 patient. Therefore, when an abnormal chest $\mathrm{x}$-ray finding is made for patients with HNCs, the lesions should be carefully evaluated for the possibility of development of second primary lung cancer. Because the median EFT was 30.5 months, clinicians should pay close attention and screen for the development of a second primary lung cancer for at least 3 years.

When HNC occurred in the oro-hypopharynx, the suspected second primary lung cancer was detected at an advanced stage. However, when the primary site was the larynx, the suspected lung cancer stage was unrelated. Because second primary lung cancers were known to occur more frequently in patients with larynx cancer, and physicians had followed the chest $\mathrm{x}$-rays or chest CTs more closely, the lung cancer stages were variable. However, because physicians were less concerned about second primary lung cancer in patients with oro-hypopharynx cancer, follow-ups with chest $\mathrm{x}$-rays or chest $\mathrm{CT}$ scan were done less frequently. As a result, the detection of a suspected lung cancer might have been delayed for the more advanced stages.

By multivariable analysis, no clinical factors were found to be statistically significant for the occurrence of a second primary lung cancer (Table 5). However, this is possibly attributable to the small number of patients with suspected second primary lung cancers and to the rather short follow-up period.
If we were to continue the study, we might possibly obtain statistically significant results.

The overall survival for patients with suspected second primary lung cancer was not different from those patients with HNC only $(p=0.430)$. However, when the EFT was excluded from the analysis for overall survival for suspected second primary lung cancer, the adjusted overall survival of suspected second primary lung cancer decreased sharply to 16 months ( $p$ $<0.001)$. This was because most of the suspected lung cancers were already at advanced stages when they were first detected by chest $\mathrm{x}$-ray. Early stage lung cancers that are detected in HNC patients can be treated with curative resections, which improves their survival. Thus, we can see the importance of early detection of second primary lung cancer in patients with primary HNCs.

The overall survival of patients with HNC has improved only minimally over the past 50 years despite advances in therapy (23). The development of second primary cancers in patients with HNCs is, in part, responsible for the failure to improve the survival rate (16). This is especially true when the second primary malignancy is lung cancer, as the treatment for lung cancer is difficult and the survival rates for advanced lung cancer are low. Therefore, early detection of second primary lung cancers that occur in HNC patients is important.

For early detection of lung cancer, we performed chest x-rays at regular intervals for HNC patients. In many patients, old pulmonary tuberculosis lesions or tiny, indeterminate nodules were detected as the initial chest $\mathrm{x}$-ray abnormalities. Because pulmonary tuberculosis is endemic to Korea and because chest CT scans are expensive, we did not routinely perform chest CT scans for patients with old pulmonary tuberculosis or indeterminate nodules. This may have caused us to overlook several observed indeterminate nodules or opacities that later developed into advanced lung cancers. If we had been able to routinely perform chest CT scans when the abnormalities were first detected on chest $\mathrm{x}$-ray, we could have found several second primary lung cancers at earlier stages. Therefore, we advise physicians to perform CT scans if any suspicious lesions are detected on the chest x-rays of patients with HNCs.

Metachronous secondary esophageal cancers were detected in 3 patients at our hospital (data not shown). Choy et al (24) reported that approximately $63 \%$ of second primary tumors for HNC patients in Hong Kong occurred in the esophagus. However, at our hospital, only 3 cases were esophageal cancers. Because most esophageal cancers are detected at an advanced stage, treatment can be very difficult. Therefore, clinicians should consider performing routine endoscopic examination for patients with HNC (24).

\section{CONCLUSION}

Patients with HNCs are at high risk for developing suspected second primary lung cancers. The statistically significant clinical risk factors for development of suspected second primary lung cancer for patients with primary HNC include oro-hypopharynx and larynx primary sites, squamous cell carcinoma and abnormal findings on chest $\mathrm{x}$-ray at the initial diagnosis. Therefore, advanced diagnostic tools, such as chest CT or PET CT scan, 
should be applied for early detection of second primary lung cancer especially when the origins of HNC are the oro-hypopharynx or larynx, the cell type is squamous cell carcinoma, and initial chest $\mathrm{x}$-ray findings are abnormal.

\section{REFERENCES}

1. Lefebvre JL. Current clinical outcomes demand new treatment options for SCCHN. Ann Oncol. 2005;16(Suppl 6):vi7-12.

2. Silverman S Jr, Gorsky M, Greenspan D. Tobacco usage in patients with head and neck carcinomas: a follow-up study on habit changes and second primary oral/oropharyngeal cancers. J Am Dent Assoc. 1983;106:33-5.

3. Dhooge IJ, De Vos M, Van Cauwenberge PB. Multiple primary malignant tumors in patients with head and neck cancer: results of a prospective study and future perspectives. Laryngoscope. 1998;108:250-6.

4. Leon X, Ferlito A, Myer CM 3rd, Saffiotti U, Shaha AR, Bradley PJ, et al. Second primary tumors in head and neck cancer patients. Acta Otolaryngol. 2002;122:765-78.

5. Jones AS, Morar P, Phillips DE, Field JK, Husband D, Helliwell TR. Second primary tumors in patients with head and neck squamous cell carcinoma. Cancer. 1995;75:1343-53.

6. Kuriakose MA, Loree TR, Rubenfeld A, Anderson TM, Datta $\mathrm{RV}$, Hill $\mathrm{H}$, et al. Simultaneously presenting head and neck and lung cancer: a diagnostic and treatment dilemma. Laryngoscope. 2002;112:120-3.

7. Atabek U, Mohit-Tabatabai MA, Raina S, Rush BF Jr, Dasmahapatra KS. Lung cancer in patients with head and neck cancer. Incidence and long-term survival. Am J Surg. 1987; 154:434-8.

8. Ritoe SC, Krabbe PF, Jansen MM, Festen J, Joosten FB, Kaanders JH, et al. Screening for second primary lung cancer after treatment of laryngeal cancer. Laryngoscope. 2002;112: 2002-8.

9. Leipzig B. Bronchoscopy in the staging and evaluation of head and neck carcinoma. Ann Otol Rhino1 Laryngol. 1983;92: 373-6.

10. Ravenel JG, Costello P, Silvestri GA. Screening for lung cancer. AJR Am J Roentgenol. 2008;190:755-61.

11. Loh KS, Brown DH, Baker JT, Gilbert RW, Gullane PJ, Irish
JC. A rational approach to pulmonary screening in newly diagnosed head and neck cancer. Head Neck. 2005;27:990-4.

12. Bayraktaroglu S, Savas R, Basoglu OK, Cakan A, Mogulkoc $\mathrm{N}$, Cagirici U, et al. Dynamic computed tomography in solitary pulmonary nodules. J Comput Assist Tomogr. 2008;32: 222-7.

13. Thompson L. World Health Organization classification of tumours: pathology and genetics of head and neck tumours. Ear Nose Throat J. 2006;85:74.

14. Gluckman JL, Crissman JD, Donegan JO. Multicentric squamous-cell carcinoma of the upper aerodigestive tract. Head Neck Surg. 1980;3:90-6.

15. Rafferty MA, O'Dwyer TP. Secondary primary malignancies in head and neck squamous cell carcinoma. J Laryngol Otol. 2001;115:988-91.

16. Vikram B. Changing patterns of failure in advanced head and neck cancer. Arch Otolaryngol. 1984;110:564-5.

17. Billroth T. Die allgemeine chirurgiche pathologie und therapie in 51 varessungen: ein handbuch fur studirende und artz. G Riemer, Berlin 1889.

18. Warren S, Gates O. Multiple primary malignant tumor: a survey of the literature and a statistical study. Am J Cancer. 1932;16:1358-414.

19. Slaughter DP, Southwick HW, Smejkal W. Field cancerization in oral stratified squamous epithelium; clinical implications of multicentric origin. Cancer. 1953;6:963-8.

20. Wu X, Hu Y, Lippman SM. Upper aerodigestive tract cancers. In: Neugut AI, Meadows AT, Robinson E (eds). Multiple primary cancers. Philadelphia, Lippincott: Williams \& Wilkins; 1999. p. 319-46.

21. Scelo G, Boffetta P, Corbex M, Chia KS, Hemminki K, Friis $\mathrm{S}$, et al. Second primary cancers in patients with nasopharyngeal carcinoma: a pooled analysis of 13 cancer registries. Cancer Causes Control. 2007;18:269-78.

22. Hsieh WC, Chen YM, Perng RP. Temporal relationship between cancers of the lung and upper aerodigestive tract. Jpn J Clin Oncol. 1997;27:63-6.

23. Larson JT, Adams GL, Fattah HA. Survival statistics for multiple primaries in head and neck cancer. Otolaryngol Head Neck Surg. 1990;103:14-24.

24. Choy AT, van Hasselt CA, Chisholm EM, Williams SR, King WW, Li AK. Multiple primary cancers in Hong Kong Chinese patients with squamous cell cancer of the head or neck. Cancer. 1992;70:815-20. 